56 Revista Latinoamericana de la Papa 20 (1): 56-65

ISSN: $1853-4961$

http://www.papaslatinas.org/ojs/index.php/index/oai

\title{
Análisis de la distinción, homogeneidad y estabilidad (DHE) de nuevos cultivares de papa (Solanum tuberosum L.) en Bolivia
}

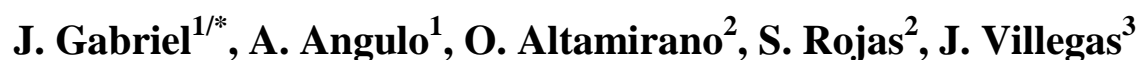

Recibido: 11/02/2016

Aceptado: 01/04/2016

Accesible en línea: Junio 2016

\section{Resumen}

El Instituto Nacional de Innovación Agropecuaria y Forestal (INIAF) de Bolivia otorga la certificación para el registro de los nuevos cultivares, siguiendo las directrices de la Unión Internacional para la Protección de las Obtenciones Vegetales (UPOV). Un cultivar puede ser registrado si cumple con los requisitos de ser diferente, homogéneo y estable (DHE) a demás de ser nueva. El objetivo de este trabajo fue determinar si cuatro cultivares de Solanum tuberosum L. cumplen con el requisito de ser DHE, necesario para ser registrados en el Registro Nacional de Variedades. Los ensayos fueron realizados cinco años de 2010 a 2014 en Cochabamba, Bolivia. La unidad experimental fueron parcelas simples de 50 plantas. Los cultivares candidatos a ser registrados fueron: 'Puka Huaycha', 'Pafrita', 'Pinker' y 'Jatun Puka'. Se incluyeron dos cultivares de uso público: 'Huaycha' y 'Desiree'. Se realizó la descripción varietal de cada cultivar en base a 32 características morfofenológicas de planta, tallo, hoja, folíolo, brote y tubérculo. Se realizó la descripción varietal de cada cultivar. Las características que mejor diferenciaron a los cultivares fueron: forma de tubérculo, color de pulpa y profundidad de yemas. Los resultados indicaron que los cultivares a ser registrados fueron diferentes a los de uso público, además presentaron homogeneidad y se mantuvieron estables en los cinco años de evaluación. Por lo tanto, los cuatro cultivares estudiados cumplen con los requisitos de DHE establecidos para la obtención del registro correspondiente.

Palabras clave adicionales: ensayo DHE, Solanum tuberosum, registro de cultivares, semillas.

\section{Analysis of the Distinctness, Uniformity and Stability (DUS) of new Cultivars of Potato (Solanum tuberosum L.) in Bolivia}

The National Institute of Agriculture, Livestock and Forestry (INIAF) of Bolivia grants titles for new cultivars, following the guidelines of the International Union for the Protection of New Varieties of Plants (UPOV). A cultivar can be protected if it meets the requirement of being different, uniform and stable (DUS), además de ser nueva. The aim of this work was to determine whether four Solanum tuberosum L. cultivars were DUS so as to be eligible to be protected. The trials were conducted during 2010 to 2014 in Cochabamba, Bolivia. The experimental units were 50 plants'- plots. The candidates to be protected were: 'Puka Huaycha', 'Pafrita', 'Pinker' and 'Jatun Puka'. The study also included two cultivars of public use: 'Huaycha' and 'Desiree'. There were studied 32 morphological and phenological

\footnotetext{
*Autor para correspondencia: Correo electrónico: j.gabriel@ proinpa.org

${ }^{1}$ Fundación PROINPA, Casilla 4285, Cochabamba, Bolivia.

${ }^{2}$ Instituto Nacional de Innovación Agropecuaria y Forestal (INIAF), Cochabamba, Bolivia.

${ }^{3}$ Instituto Nacional de Innovación Agropecuaria y Forestal (INIAF), Sucre, Bolivia.
} 
characteristics of plant, stem, leaf, leaflets, bud and tuber. A varietal description was recorded for each cultivar. The characteristics that best differentiated the cultivars were: shape of tuber, flesh color and eye depth. The results indicate that the candidate cultivars are different from those of public use, and that they were homogeneous and stable in five years of evaluation. Therefore, the four cultivars studied meet the DUS requirements to obtain the correspondent title.

Adittional key words: DUS test, Solanum tuberosum, property of varieties, seeds

\section{Introducción}

Bolivia se incluyó al convenio de la Unión Internacional para la Protección de las Obtenciones Vegetales (UPOV) el 22 de marzo de 1999 (OMPI, 2015). La importancia de registrar una variedad en el Registro Nacional de Variedades, está mencionada en el artículo 15 de la Norma General Sobre Semillas de Especies Agrícolas, aprobada en la Resolución de Directorio del INIAF $\mathrm{N}^{\mathrm{o}}$ 02/2009, mediante la cual se establece que "Ingresarán al proceso de certificación de semillas únicamente aquellas variedades que se encuentren inscritas en el Registro Nacional de Variedades" (INIAF-DNS, 2012). El Registro Nacional de Variedades establece en el párrafo 6.2 del anexo, que para la protección de un cultivar, este debe satisfacer los criterios de distinción, homogeneidad, estabilidad, novedad y denominación adecuada conforme a la UPOV (1996). Para determinar si un cultivar es diferente, homogéneo y estable, se realizan una serie de ensayos comúnmente denominados DHE, además de cumplir con las pruebas de Valor Agronómico.

La institución responsable de examinar las solicitudes de registro, realizar las comprobaciones técnicas y otorgar los títulos son certificados de registro a los cultivares es el Instituto Nacional de Innovación Agropecuaria y Forestal de Bolivia (INIAF), según el artículo $10 \mathrm{del}$ R.M. 045 de Marzo de 2005 (INIAFRNVyVP, 2014). En el INIAF se realizan todos los años una gran cantidad de ensayos de DHE para otorgar los títulos certificados de registro de cultivares (INIAF-RNVyVP, 2014).
Para obtener el registro a un cultivar nuevo, este debe diferenciarse en al menos una característica relevante como forma de tubérculo, color de pulpa, color de piel, profundidad de yemas, etc. de los demás cultivares conocidos y además, estas deben mantenerse inalterables en las sucesivas generaciones y cumplir con la condición de ser nueva (UPOV, 1996; UPOV, 2002).

En Bolivia la papa ocupa el primer lugar entre los tubérculos cultivados con una superficie aproximada de 140.000 ha de cultivo e involucra aproximadamente a 200.000 agricultores que representan el 30 a $40 \%$ del total de agricultores del país (Blajos et al. 2007, Zeballos et al. 2009; Gabriel, 2010). Es la principal fuente de alimentación e ingresos en Bolivia (Estrada et al. 1994, Fernández-Northcote et al. 1999), y se conoce que 114 municipios del país han priorizado la papa como el principal cultivo para la alimentación (Zeballos et al. 2009). La producción es aproximadamente de 750 mil toneladas al año, lo cual representa entre 300 a 600 millones de bolivianos (Blajos et al. 2007, Zeballos et al. 2009). Cuenta además, con 31 cultivares registrados de papa en el Registro Nacional de Variedades y Variedades Protegidas (INIAF-RNVyVP, 2014).

El objetivo de este trabajo fue determinar si cuatro cultivares de Solanum tuberosum L. cumplen con los requisitos de ser Diferentes, Homogéneos y Estables, necesarios para otorgarles el registro y realizar la descripción varietal detallada de cuatro cultivares en Bolivia. 


\section{Materiales y métodos}

Se evaluó cuatro cultivares mejorados de papa resistentes al tizón (Puka Huaycha, Pafrita, Pinker y Jatun Puka) y dos testigos (Desiree y Huaycha), durante cinco años en parcelas simples en las zonas de Chullchunqani en la provincia Carrasco (Cochabamba) a $17^{\circ} 32^{\prime}$ de latitud sur, $65^{\circ} 22^{\prime}$ de longitud oeste y altura promedio de $3086 \mathrm{msnm}$; Compañía Pampa, San Isidro y Piusilla en el municipio de Morochata en la provincia Ayopaya a $17^{\circ} 14^{\prime}$ de latitud sur, $65^{\circ} 31^{\prime}$ de longitud oeste y altura promedio de 3089 msnm y Primera Candelaria en el municipio de Colomi en la provincia Chapare a $17^{\circ} 20^{\prime}$ de latitud sur, $65^{\circ} 51^{\prime}$ de longitud oeste y altura promedio de $3200 \mathrm{msnm}$. La información general del origen y desarrollo de estos cultivares están descritas en Gabriel et al. (2006), Gabriel et al. (2008) y Gabriel (2010) y las características morfológicas, agronómicas, de resistencia, características organolépticas y los lugares de evaluación, están descritas en Gabriel et al. (2011). La metodología utilizada fue la que establece las directrices para la ejecución de exámenes de DHE (TG/23/6) de papa de la UPOV (UPOV, 2004) y los descriptores recomendados por el INIAF-DNS-UFRS (2013).

La procedencia de los cuatro cultivares mejorados y de los dos de uso público que se sembraron se observan en Tabla 1.

Tabla 1. Procedencia y obtentor de cuatro cultivares de papa a ser registrados evaluados junto a dos cultivares de uso público.

\begin{tabular}{|c|c|c|c|c|}
\hline Cultivar & Obtentor & Institución & Origen & Referencias \\
\hline Puka Huaycha & Julio Gabriel & Fundación PROINPA & Bolivia & $\begin{array}{l}\text { Gabriel et al. (2010), } \\
\text { INIAF-RNVyVP, } \\
\text { (2014) }\end{array}$ \\
\hline Pafrita & Julio Gabriel & Fundación PROINPA & Bolivia & Gabriel et al. (2010) \\
\hline Pinker & Julio Gabriel & Fundación PROINPA & Bolivia & Gabriel et al. (2010) \\
\hline Jatun Puka & CIP & CIP & Perú & $\begin{array}{l}\text { Gutiérrez-Rosales et } \\
\text { al. (2007) }\end{array}$ \\
\hline Huaycha* & & & Bolivia & $\begin{array}{l}\text { INIAF-RNVyVP, } \\
\text { (2014) }\end{array}$ \\
\hline Desiree* & & & Holanda & $\begin{array}{l}\text { INIAF-RNVyVP, } \\
\text { (2014) }\end{array}$ \\
\hline
\end{tabular}

*: Cultivares de uso público

Para los ensayos la siembra fue realizada en forma manual. La unidad experimental fueron parcelas de cinco surcos con 10 plantas cada uno, distanciados entre sí a $0,80 \mathrm{~m}$ y $0,30 \mathrm{~m}$ entre plantas. Los tratamientos fueron agrupados según momento de madurez y color de la piel del tubérculo.

La fertilización básica se realizó inmediatamente después de la siembra en forma manual a chorro continuo. Se utilizó estiércol de bovino y Fosfato diamónico (18-46-00) a una dosis de 5 t.ha ${ }^{-1}$ y 0,26 t.ha $^{-1}$ respectivamente. También se aplicó
Urea (46-00-00) en el momento del aporque en una dosis de 72,07 kg.ha- ${ }^{-1}$. Las dosis de los fertilizantes fueron cuantificadas con balanzas tipo Ferton Professional ACS - B y analítica Acculab AL 64.

Durante el desarrollo del cultivo se controló las malezas en forma manual. Para el control de plagas como gusanos cortadores (Agrostis sp.), se aplicó el insecticida, Lambda-cihalotrina a una dósis de 15 cc/20 L. Al $80 \%$ de emergencia se aplicó un fungicida sistémico (Metalaxyl), a los siete días se alternó con un fungicida 
de contacto (Mancozeb), para prevenir las enfermedades como el tizón tardío (Phytophthora infestans), debido al periodo de lluvias.

Las características en campo fueron: altura de planta, estructura del follaje, porte de la planta y pigmentación antociánica del tallo.

Se recolectaron hojas completamente desarrolladas para el análisis de tamaño de hoja, intensidad del color verde, pigmentación antociánica de la nervadura central, presencia de folíolos secundarios, tamaño de los folíolos laterales, relación ancho y largo de los folíolos laterales, frecuencia de coalescencia del folíolo terminal y laterales, ondulación del borde del foliolo, pubescencia en el haz, profundidad de las nervaduras y brillo del haz del folíolo (Huamán et al., 1977; UPOV, 1996; 2002; 2004 ).

La cosecha de los ensayos se realizó cuando todos los cultivares llegaron al momento óptimo de cosecha, que se determina cuando se alcanza el máximo de materia seca en el tubérculo, y existe disminución de los azúcares totales, lo que se dio conjuntamente con el fortalecimiento de la piel (Crisci y Vilaró, 1993; Boschi e Ibarra, 2012). Las características de tubérculo evaluados en almacén y fueron: dormancia, forma, profundidad de las yemas, color de la base de las yemas, color de la piel y color de la pulpa. Posteriormente al análisis de los tubérculos se evaluaron las características de brote, según las directrices de exámenes de la UPOV (UPOV, 2004). Las características de brote que se estudiaron fueron: coloración antociánica, intensidad de la pigmentación antociánica en la base, pubescencia en la base, tamaño, forma, tamaño del extremo en relación a la base, porte del extremo, pubescencia del extremo, pigmentación antociánica del extremo, número de radículas y longitud de las ramificaciones laterales.

\section{Resultados y discusión}

Todos los cultivares se diferenciaron en al menos una característica de los otros cultivares candidatos a ser protegidos y los de uso público. En todas las localidades y años, las características evaluadas para cada uno de los cultivares fueron lo suficientemente homogéneas dentro de cada cultivar y las características relevantes se mantuvieron estables, cumpliendo así con los requisitos de homogeneidad y estabilidad.

En la Tabla 2 se presentan las características de planta y hoja menos y más influenciadas por las condiciones ambientales. Las menos influenciadas fueron: estructura del follaje, porte de la planta y pigmentación antociánica del tallo (UPOV, 2004). Por otra parte, aquellas con una diferenciación varietal marcada en las condiciones ambientales de Bolivia fueron: pigmentación antociánica de la nervadura central, relación ancho y largo de los folíolos laterales y ondulación del borde. 
Tabla 2. Características de planta, tallo, hoja, folíolos laterales y folíolos de cuatro cultivares de papa mejorados y dos de uso público.

\begin{tabular}{llllllll}
\hline Cultivar & $\begin{array}{l}\text { Estructura } \\
\text { del follaje }\end{array}$ & Porte & $\begin{array}{l}\text { Pigmentación } \\
\text { antociánica }\end{array}$ & $\begin{array}{l}\text { Pigmentación } \\
\text { Antociánica } \\
\text { de }\end{array}$ & $\begin{array}{l}\text { Relación: } \\
\text { nervadura } \\
\text { ancho } \\
\text { longitud }\end{array}$ & $\begin{array}{l}\text { Ondulación } \\
\text { del borde }\end{array}$ \\
\hline $\begin{array}{l}\text { Puka } \\
\text { Huaycha } \\
\text { Pafrita }\end{array}$ & Intermedio & Erecto & Fuerte & Media & Media & Fuerte \\
Intermedio & Semierecto & Ausente & $\begin{array}{l}\text { Ausente } \\
\text { muy débil }\end{array}$ & o & Media & Media \\
Jatun Puka & Intermedio & Semierecto & Ausente & $\begin{array}{l}\text { Ausente } \\
\text { muy débil }\end{array}$ & o & Media & Media \\
Intermedio & Erecto & Media & $\begin{array}{l}\text { Ausente } \\
\text { muy débil }\end{array}$ & o & Media & Media \\
Desiree* & Intermedio & Erecto & Fuerte & $\begin{array}{l}\text { Media } \\
\text { Ausente } \\
\text { muy débil }\end{array}$ & o & Media & Fuerte \\
\hline
\end{tabular}

*: Cultivares de uso público.

De acuerdo a las características analizadas en la Tabla 2, el cultivar mejorado Puka Huaycha y el de uso público Huaycha difieren de los demás cultivares en el porte de la planta, la pigmentación antociánica del tallo, la pigmentación antocíanica de la nervadura central y la ondulación de los bordes. Los cultivares Puka Huaycha y Huaycha expresaron en el tallo una pigmentación antociánica fuerte $\mathrm{y}$ pigmentación antociánica media para la nervadura central de la hoja media. Por otra parte, los demás cultivares expresaron pigmentación media o ausente en el tallo y ausente o muy débil en la nervadura central de la hoja.

El porte de la planta es una característica sencilla de identificar en campo porque se observa la disposición de las ramas secundarias respecto al tallo principal, por lo tanto, presenta baja subjetividad, los tres cultivares semierectos rastreros Pafrita, Pinker y Desiree se diferenciaron muy fácilmente de los cultivares erectos Puka Huaycha y Huaycha.

La relación entre el ancho y la longitud de los folíolos laterales es una medida objetiva cuya determinación se realiza con medición y se califica con la escala estrecha, media $y$ ancha. No hubo diferencia para ninguno de los cultivares, todos tuvieron una relación media.

En la Tabla 3 se presentan las características de brote menos influenciadas por las condiciones ambientales en que se realizó el ensayo (UPOV, 2004). 
Tabla 3. Características de brote de cuatro cultivares mejorados y dos cultivares de uso público.

\begin{tabular}{|c|c|c|c|c|c|}
\hline Cultivar & $\begin{array}{l}\text { Intensidad de } \\
\text { pigmentación } \\
\text { antociánica en } \\
\text { la base }\end{array}$ & $\begin{array}{l}\text { Pubescencia } \\
\text { en la base }\end{array}$ & Forma & $\begin{array}{l}\text { Tamaño } \\
\text { extremo en } \\
\text { relación con } \\
\text { la base }\end{array}$ & $\begin{array}{l}\text { Número de } \\
\text { radículas }\end{array}$ \\
\hline Puka Huaycha & Fuerte & Fuerte & Cónica & Medio & Medio \\
\hline Pafrita & Media & Media & Cónica & Pequeño & Medio \\
\hline Pinker & Media & Media & Cónica & Pequeño & Medio \\
\hline Jatun Puka & Media & Media & Cónica & Medio & Medio \\
\hline Huaycha* & Fuerte & Fuerte & Cónica & Medio & Medio \\
\hline Desiree* & Media & Media & Cónica & Pequeño & Medio \\
\hline
\end{tabular}

*: Cultivares de uso público.

Los cultivares 'Puka Huaycha' y 'Huaycha' se diferenciaron en la intensidad de la pigmentación antociánica y pubescencia de la base de los brotes, las cuales fueron fuertes respecto de las de los cultivares 'Pafrita', 'Pinker' y 'Jatun Puka'.

Se debe mencionar, que el cultivar 'Jatun Puka' descrito en este artículo, es el mismo que fue registrado como 'Unica' en Perú (Gutiérrez-Rosales et al., 2007). En Colombia se registró una variedad denominada 'ICA Unica' (UNC, 2015); pero diferente al mencionado. Debido a lo indicado y para evitar confusiones las normas específicas de identificación de semilla en Bolivia, no permite el registro con el mismo nombre (INIAF-RNVyVP,
2014), por lo que se decidió denominarla como 'Jatun Puka'.

En las características de brote todos los cultivares se diferenciaron de forma clara en al menos una característica. La forma del brote es una característica pseudocualitativa en la que los extremos de la escala son esférica y cilíndrica estrecha. Todos los cultivares presentaron forma de brote cónica. En los cultivares 'Puka Huaycha' y 'Huaycha' el tamaño del extremo del brote en relación con la base fue medio, en cambio en los cultivares 'Pafrita, 'Pinker' y 'Jatun Puka' fue pequeño.

En la Tabla 4 se presentan las características de tubérculo que mejor diferencian a los cultivares.

Tabla 4. Características de tubérculo de cuatro cultivares de papa mejorados y de dos cultivares de uso público.

\begin{tabular}{|c|c|c|c|c|c|}
\hline Cultivar & Forma & $\begin{array}{l}\text { Profundidad } \\
\text { de las yemas }\end{array}$ & $\begin{array}{l}\text { Color de la } \\
\text { base de las } \\
\text { yemas }\end{array}$ & Color de la piel & $\begin{array}{lll}\text { Color } & \text { de } & \text { la } \\
\text { pulpa } & & \\
\end{array}$ \\
\hline Puka Huaycha & Redonda & Profundas & Blanco & Parcialmente rojo & Blanco \\
\hline Pafrita & Ovalada & $\begin{array}{l}\text { Muy poco } \\
\text { profundas }\end{array}$ & Rojo & Rojo & Crema \\
\hline Pinker & Ovalada largo & $\begin{array}{l}\text { Muy poco } \\
\text { profundas }\end{array}$ & Rojo & Rojo & Crema \\
\hline Jatun Puka & Ovalada largo & $\begin{array}{l}\text { Muy poco } \\
\text { profundas }\end{array}$ & Rojo & Rojo & Crema \\
\hline Huaycha* & Redonda & Muy Profundas & Blanco & Parcialmente rojo & Blanco \\
\hline Desiree* & Ovalada & Muy profundas & Rojo & Rojo & Amarillo claro \\
\hline
\end{tabular}

*: Cultivares de uso público.

Los cultivares 'Puka Huaycha' y 'Huaycha presentan forma de tubérculo redonda; por otra parte, las variedades 'Pafrita' y 'Jatun
Puka' tienen forma ovalada y las variedades 'Pinker' y 'Desiree' forma ovalada larga. 'Puka Huaycha' y 'Huaycha 
se diferencian de las demás también en la profundidad y en el color de la base de las yemas, sumándose el color de la piel y la intensidad de la pigmentación antociánica en la base del brote. Aunque esta diferencia es subjetiva.

La forma, el color de la pulpa y el color de la piel del tubérculo son características relevantes para diferenciar e identificar cultivares. 'Pinker' y 'Jatun Puka' tiene el tubérculo ovalado alargado, en cambio 'Pafrita' y 'Desiree' son ovalados. El color de la base de las yemas es una característica poco influenciada por las condiciones ambientales en las que se realiza el ensayo (UPOV, 2004).

Los cultivares que presentaron mayores similitudes en la mayoría de las características morfofenológicas fueron 'Puka Huaycha' y 'Huaycha' que solamente se diferenciaron en la profundidad de las yemas, profundas $y$ muy profundas respectivamente; 'Pafrita' y 'Pinker' que se diferenciaron en la forma del tubérculo, ovalada y ovalada larga respectivamente y 'Desiree'y 'Pinker'en el color de la pulpa, crema y amarillo claro respectivamente.

El color de la piel y el color de la pulpa son características que no varían con los cambios en las condiciones ambientales (UPOV, 2004) y además, son importantes para los agricultores en la selección de un cultivar. Para el color de piel el rango de variación estuvo entre parcialmente rojo: 'Puka Huaycha' y 'Huaycha', rojo: 'Pafrita', 'Pinker' y 'Jatun Puka'. En cambio, la amplitud de rango para la característica de color de pulpa fue mayor, hubo cultivares de color blanco, amarillo claro y crema. El cultivar 'Jatun Puka' descrita en el presente trabajo concuerda con lo reportado por Gutiérrez-Rosales et al. (2007).

Se realizó la descripción varietal de los cuatro cultivares estudiados, estas se encuentran disponibles en el Registro Nacional de Variedades y Variedades protegidas del INIAF. A modo de ejemplo en el Cuadro 5 se presentan las características varietales de los cultivares: 'Pinker' y 'Desiree'. 
Tabla 5. Descripción varietal de dos cultivares de S. tuberosum.

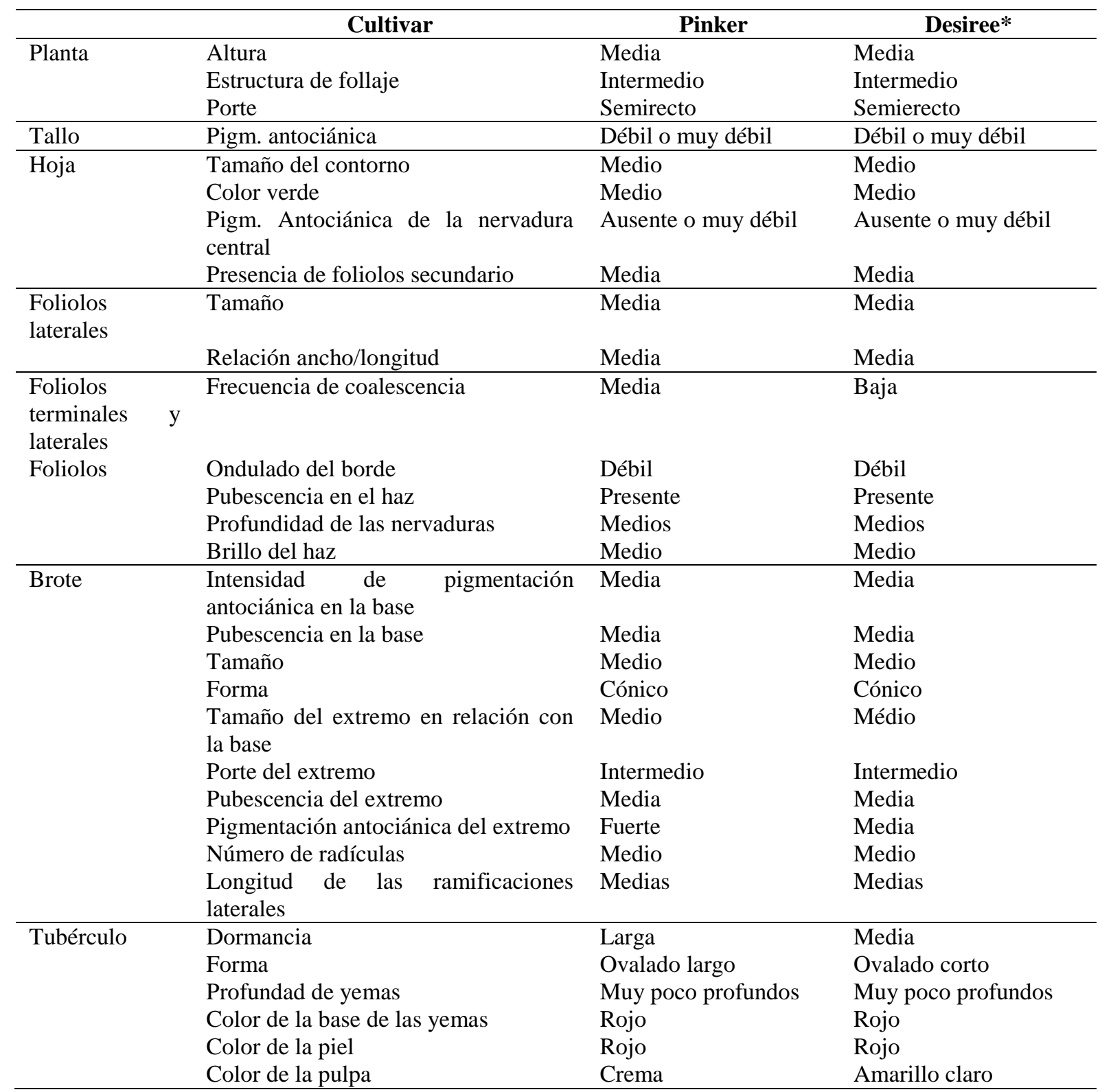

*: Cultivar de uso público.

Los cultivares disponibles en el mercado para producción en su mayoría presentan color de piel rojo o parcialmente rojo como 'Huaycha' y 'Desiree' respectivamente. Con respecto al color de pulpa, la mayoría de los cultivares que predominan en el mercado son de color blanco; sin embargo, se han incrementado las solicitudes de protección con cultivares que presentan colores de pulpa no comunes, como pueden ser 'Pinta Boca' y 'Candelero' que son violeta y rojo respectivamente (Cadima et al., 2004). Finalmente mencionar que todos los cultivares evaluados tienen una resistencia básica al tizón tardío (Phytophthora infestans) y virus (PVY y PVX) (Veramendi et al., 2011; GutiérrezRosales et al., 2007). Algunos además tienen combinaciones de resistencias a otros factores bióticos como nematodos (Nacobbus aberrans, Globodera sp.) y verruga (Synchytrium endobioticum); y otras escapan al efecto de las sequía u heladas (Pafrita, Pinker) (Gabriel et al. 2011) y con características de semiprecocidad (Puka Huaycha). 


\section{Agradecimientos}

Este trabajo fue desarrollado por la Fundación PROINPA, en el sub-proyecto "Desarrollo de variedades con tolerancia a factores bióticos" ejecutado en el marco del Programa Nacional de Papa del INIAF. Se realizó con ayuda financiera del Banco Mundial y el proyecto Mercados Rurales de Swisscontact. Las opiniones expresadas en el mismo no reflejan necesariamente la opinión oficial de estas instituciones.

\section{Conflictos de interés}

Esta investigación fue desarrollada por la Fundación PROINPA y no presenta conflictos de interés.

\section{Referencias citadas}

Boschi, F.; Ibarra, M. 2012. Análisis de la distinción, homogeneidad y estabilidad (DHE) de nuevos cultivares de papa (Solanum tuberosum L.) en Uruguay. Agrociencia, Uruguay - 16 (2):74-81.

Blajos, J.; Amaya, N.; Gandarillas, A. 2007. ¿Cuán importante es la papa para los bolivianos?. Revista de Agricultura, Bolivia 40 (59):7-9

Cadima, X., Gonzáles, R., Almanza, J.; García, W.; Terrazas, F. 2004. Catálogo de variedades de papa locales de papa y oca en la zona de Candelaria. Fundación PROINPA- CIP-COSUDE, Cochabamba, Bolivia. $112 \mathrm{p}$.

Crisci, C.; Vilaró, F. 1993. Aportes tecnológico para el cultivo de papa. Montevideo : INIA. 34p. (Boletín de divulgación 32).

DIEA. 2010. Anuario Estadístico Agropecuario 2009. Montevideo : MGAP. $168 \mathrm{p}$.

Estrada, N.; Fernández-Northcote, E.; Carrasco, E.; Navia. O. 1994. Mejoramiento genético para resistencia a enfermedades y plagas de la papa en Bolivia. p. 86-89. L.H.M. Broers (Ed.) en: Resistencia duradera en cultivos alto andinos, Memorias del 1er taller sobre Resistencia Duradera en Cultivos Alto Andinos. 30 de mayo-3 de junio de 1994. Quito, Ecuador

Fernández-Northcote, E.; Navia, O.; Gandarillas, A. 1999. Bases de las estrategias de control químico del tizón tardío de la papa desarrolladas por PROINPA en Bolivia. Revista Latinoamericana de la papa 11:1- 25 p.

Gabriel, J.; Herbas, J.; Salazar, M.; López, J.; Villarroel, J.; Cossio, D. 2006. Participatory plant breeding: A new challenge in the generation and appropriation of potato varieties by farmers in Bolivia. PRGA Consultative Group on International Agricultural Research (CGIAR), Fundacion PROINPA (Working Document no. 22).

Gabriel, J.; Vallejos, J.; Coca, C.; López, J.; Escobar, F.; Villarroel, E.; Villarroel, J. 2008 Agricultores generan sus propias variedades de papa en colaboración con los fitomejoradores de PROINPA: Una experiencia exitosa en Morochata, Bolivia. Revista de Agricultura (Bolivia) 42 (60): 26-30.

Gabriel, J. 2010. Documento marco: Estrategias y perspectivas del mejoramiento genético de papa (Solanum tuberosum L.) en Bolivia. Fundación PROINPA, Cochabamba, Bolivia. 60 p.

Gabriel, J.; Pereira, R.; Gandarillas, A. 2011. Catálogo de nuevas variedades de papa en Bolivia. Fundación PROINPA, Cochabamba, Bolivia. 55 p.

Gutiérrez-Rosales, R.O.; Espinoza-Telles, J.A.; Bonierbale, M. 2007. : variedad Peruana para mercado fresco y papa frita con tolerancia y resistencia para condiciones climáticas adversas. Revista Latinoamericana de la Papa 14(1): 41-50.

Huamán, Z.; William J.T.; Salhuana, W.; Vincent, L. 1977. Descriptors for the 
cultivated potato. Intenational board for Plant genetic Resources (IBPRG), Rome, Italy. $45 \mathrm{p}$.

INASE. 2010. Registro de propiedad de cultivares [En línea]. Disponible en: http://www.inase.org.uy/especiesCultivares /Default.aspx. Consulta: Enero 2016.

INIAF-DNS. 2012. Registro nacional de variedades y variedades protegidas. Marco Normativo Institucional. MDRyT, La Paz, Bolivia. 48 p.

INIAF-DNS-UFRS. 2013. Descriptores de variedades inscritas en el Registro Nacional de Variedades. INIAF-DNSUFRS, La Paz, Bolivia. 163 p.

INIAF-RNVyVP. 2014. Registro Nacional de Variedades 2014. INIAF, RNV y VP, La Paz, Bolivia. 39 p.

OMPI. 2015. Notificación UPOV No 72. Convenio Internacional para la Protección de las Obtenciones Vegetales de 2 de diciembre de 1961, revisado en Ginebra el 10 de noviembre de 1972 y el 23 de octubre de 1978. Adhesión por la República de Bolivia. http://www.wipo.int/treaties/es/notification s/upov/treaty_upov_72.html. Consulta: Enero 2016.

UNC. 2015. ICA Unica. Grupo de Investigación de papa, Universidad Nacional de Colombia (UNC), Bogota, Colombia. [En llínea]. Disponible en: http://www.papaunc.com/catalogoExtendid o.shtml?x=40. Consulta: Febrero, 2016.
UPOV. 1996. Convenio internacional para la protección de las obtenciones vegetales. Ginebra: UPOV. 31p. (Texto oficial en español; 221).

UPOV. 2002. Introducción general al examen de la distinción, la homogeneidad y la estabilidad y la elaboración de descripciones armonizadas de las obtenciones vegetales. [En línea]. Disponible en: http:// www.upov.int/export/sites/upov/publicatio ns/es/tg rom/pdf/tg_1_3.pdf. Consulta: Enero 2016.

UPOV. 2004. Directrices para la ejecución del examen de la distinción, la homogeneidad y la estabilidad de Solanum tuberosum L. (papa). [En línea]. Disponible en: http://www.upov.int/edocs/tgdocs/es/tg023 .pdf. Consulta: Enero, 2016.

Veramendi, S.; Baldelomar, M.; Terán, A.; Gabriel, J. 2011. Marcadores moleculares asociados a genes/QTLs de resistencia para factores bióticos en nuevas variedades de papa (Solanum tuberosum L.) de Bolivia. Revista Latinoamericana de la Papa 16 (2): 209 - 232.

Vilaró, F.; Pereira, G.; Rodriguez, G. 2004. Características y recomendaciones de manejo del cultivar de papa INIA Iporá. Montevideo: INIA. 4p. (Hoja de Divulgación ; 86).

Zeballos, H.; Balderrama, F.; Condori, B.; Blajos, J. 2009. Economía de la papa en Bolivia (1998-2007). Fundación PROINPA, Cochabamba, Bolivia. 129 p. 